\title{
Unfolding and Refolding of Protein by a Combination of Ionic and Nonionic Surfactants
}

\author{
Debasish Saha, ${ }^{\dagger}$ Debes Ray, ${ }^{\dagger}$ Joachim Kohlbrecher, ${ }^{\ddagger}$ and Vinod Kumar Aswal ${ }^{*}{ }^{\dagger}$ (৫) \\ ${ }^{\dagger}$ Solid State Physics Division, Bhabha Atomic Research Centre, Mumbai 400 085, India \\ ${ }^{\ddagger}$ Laboratory for Neutron Scattering and Imaging, Paul Scherrer Institut, CH-5232 PSI Villigen, Switzerland
}

Supporting Information

ABSTRACT: The interaction of protein and surfactant yields protein-surfactant complexes which have a wide range of applications in the cosmetics, foods, and pharmaceutical industries among others. Ionic and nonionic surfactants are known to interact differently with the protein. The interplay of electrostatic and hydrophobic interactions governs the resultant structure of protein-surfactant complexes. The present study enlightens the paramount role of the hydrophobic interaction, tuned by the hydrophobic tail length of ionic surfactants, in the unfolding of anionic bovine serum albumin (BSA) protein. The unfolding of BSA in the presence of four different tail-length cationic surfactants, that is, C10TAB, C12TAB, C14TAB, and C16TAB, has been
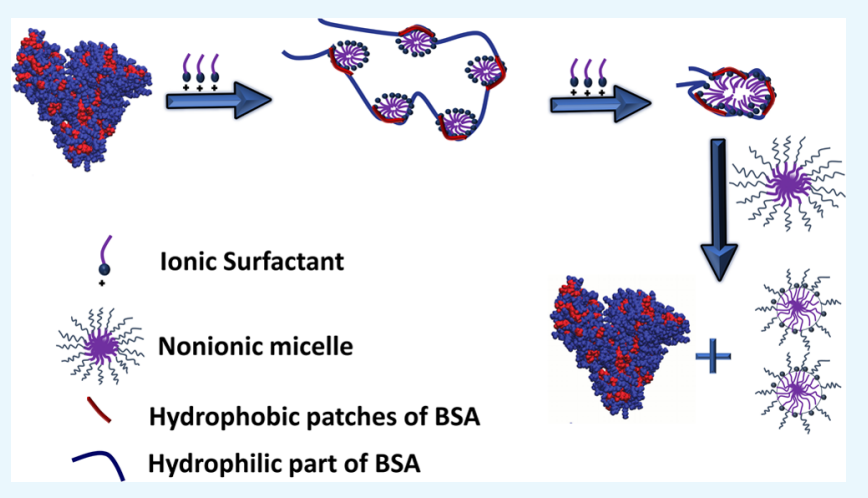
investigated by small-angle neutron scattering and dynamic light scattering. All cationic surfactants unfold the protein at a certain concentration range. The propensity of protein unfolding increases with increasing the hydrophobic tail length. The denatured structure of BSA upon addition of cationic surfactants is characterized by the random flight model representing a beads-on-a-string chain-like complex. The unfolded protein binds the surfactant micelles in the protein-surfactant cluster. The micelles get elongated with the increasing concentration of cationic surfactants, whereas the number of micelles per cluster is decreased. In the final stage, the protein-surfactant cluster merges to one large micelle with unfolded protein wrapping the micelle surface. The pathway of protein unfolding is described in terms of the changes in the micellar size, the number of micelles formed per cluster, the separation between the micelles in the cluster, the aggregation number of micelles, and the number of proteins per cluster. The protein-surfactant interaction is further examined in the presence of a nonionic surfactant, that is, C12E10. The nonionic surfactant significantly suppresses the interaction of BSA protein with ionic surfactants by forming mixed micelles. As a result of the mixed micelles formation by ionic-nonionic surfactants, the ionic surfactant moves out from the unfolded BSA protein, and this enables the protein to refold back to its native structure. The propensity of mixed micelle-driven refolding of proteins is significantly changed with changing the tail length of the ionic surfactant.

\section{INTRODUCTION}

Proteins and surfactants are among the most important entities of the soft matter, which, by combining, lead to several interesting applications in the pharmaceutical, cosmetic, and food industries. ${ }^{1-3}$ Bovine serum albumin (BSA), one of the most investigated proteins, shows a very rich phase behavior with surfactants in the aqueous solution because of their common amphiphilic nature. ${ }^{4-6}$ From the viewpoint of surface activity, both the proteins and surfactants are individually surface active. It is a firmly established fact that ionic surfactants such as sodium dodecyl sulfate (SDS) or dodecyltrimethylammonium bromide (DTAB) could denature proteins at low concentrations relative to other denaturants such as urea or guanidinium chloride. ${ }^{7-11}$ On the contrary, nonionic surfactants only play a minor role in the denaturation of some proteins. ${ }^{12,13}$ The emerging scientific interest consists of the detailed understanding of protein-surfactant inter- actions to follow the pathway of unfolding and refolding of the protein.

Ionic surfactants can interact with the protein because of the site-specific interaction of surfactants on the oppositely charged patches of the protein. ${ }^{14-16}$ On the contrary, nonionic surfactants can have only a hydrophobic interaction with the protein. ${ }^{15,17}$ The differences in the interaction of the protein with ionic and nonionic surfactants suggest that the proteinsurfactant interaction is primarily driven by their electrostatic interaction. The electrostatic interaction between the protein and an individual ionic surfactant occurs at the surface of the protein, which is usually not sufficient to perturb the native structure of the protein. ${ }^{6,18,19}$ We identify by dynamic light scattering (DLS), an optimal concentration up to which

Received: April 3, 2018

Accepted: July 10, 2018

Published: July 25, 2018 
$\mathrm{CnTAB}(n=10,12,14$, and 16) molecules are attached individually to the protein surface or stay free in the solution. Above this optimal concentration, which is known as critical aggregation concentration (CAC), surfactant molecules form micelle aggregates and initiate protein denaturation. In reality, CAC can be significantly below the critical micelle concentration $(\mathrm{cmc}){ }^{20}$ As the process of denaturation induced by ionic surfactants proceeds, the protein unfolds extensively, and thus, the internally directed hydrophobic regions become exposed on the outside of the molecule. This situation is entropically unfavorable because the hydrophobic segment of protein molecules is not stable in the aqueous environment. ${ }^{21}$ The entrapment of water leads to the micelle-like cluster formation of protein-surfactant complexes. ${ }^{7,22}$ These proteinsurfactant complexes can be described by the random flight model representing a beads-on-a-string. ${ }^{7,22,23}$ As the surfactant concentration is increased, the protein-decorated micelles start elongating, mostly in one direction, and therefore, the number of micelles per cluster is decreased. In the final stage, a large micelle with a very high aggregation number is formed, and the cluster formation disrupted. In principle, the number of proteins per micelle becomes too small to allow extension of the cluster. The unfolded protein wraps the large micelle through its surface. Thus, the interplay of electrostatic and hydrophobic interactions governs the resultant structure of the protein in the protein-surfactant complexes. There is another widely used model called the "bead-necklace" model to define the protein-surfactant complexes. ${ }^{9,24,25}$ However, the beadnecklace model does not include the scattering from the protein, which limits its use to explain the scattering from protein-surfactant complexes in the absolute scale.

In this study, we have considered tuning of the proteinsurfactant interaction to modify the resultant structure by varying the hydrophobic tail length of ionic surfactants. The work reveals that the hydrophobic interaction, which is responsible for protein unfolding, can be controlled by the hydrophobic tail length of the ionic surfactant. It is found that the protein-surfactant complexes with longer tail-length surfactants reach more rapidly the final stage of the unfolding process, where the cluster formation is disrupted. The results suggest that the propensity of protein unfolding induced by the ionic surfactant is increased with increasing the hydrophobic tail length of the ionic surfactant. Taking the benefit of the absence of the electrostatic interaction between proteins and nonionic surfactants and the presence of strong hydrophobic interaction between the ionic and nonionic surfactants, an innovative way is proposed to refold back the unfolded protein or prevent the protein unfolding. ${ }^{11,13,26,27}$ The hydrophobic interaction between the protein and the ionic surfactant, and in the ionic-nonionic surfactant mixed micelle complexes, is examined by changing the hydrophobic tail length. The dominance of the hydrophobic interaction between ionic and nonionic surfactants over the electrostatic interaction of protein and ionic surfactants interrupts the cluster formation. In the process of refolding, initially large micelles but small in number are formed at a lower nonionic surfactant concentration. The number of micelles increases, whereas the size is decreased with increasing C12E10 concentration. At a higher C12E10 concentration, the interaction of the BSA protein with ionic surfactants is completely suppressed by the ionicnonionic mixed micelle formation. The withdrawal of ionic surfactants from the protein-surfactant complexes allows them to refold back to their native structure. The propensity of mixed micelles-driven refolding of protein is significantly changed with changing the tail length of the ionic surfactant.

The interaction of BSA with different cationic surfactants with increasing hydrophobic tail length, decyltrimethylammonium bromide (C10TAB), DTAB (C12TAB), tetradecyltrimethylammonium bromide $(\mathrm{C} 14 \mathrm{TAB})$, and hexadecyltrimethylammonium bromide (C16TAB) with and without nonionic polyoxyethylene 10 lauryl ether (C12E10) is studied by small-angle neutron scattering (SANS) and DLS. The unfolding of the protein structure in the presence of cationic surfactants is examined with varying concentrations of surfactants, whereas the refolding of the same is studied in the presence of combined cationic and nonionic surfactants at varying nonionic surfactant concentrations while keeping the cationic surfactant concentration fixed. The detailed structural analysis of the above protein-surfactant complexes is reported using SANS data and supported by DLS measurements. $9,11,13,27,28$

\section{RESULTS AND DISCUSSION}

2.1. Unfolding of Protein: Role of Surfactant Tail Length. In the protein-surfactant complexes, some significant concentrations of the monomeric $\mathrm{CnTAB}$ are present, which can interact with BSA in ways that are different from the micelle formation. The surfactant molecules, which do not participate in the micelle formation, are quantified by DLS measurement by finely tuning the ionic surfactant concentration and tracking the changes in the hydrodynamic size at the same time. The interactions of $1 \mathrm{wt} \%$ BSA with differently concentrated $\mathrm{C} n \mathrm{TAB}$ are presented in Figure 1. The numbers

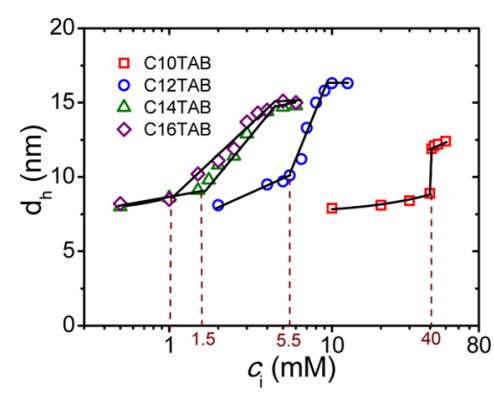

Figure 1. Hydrodynamic size variation of $1 \mathrm{wt} \%$ BSA with differently concentrated $\mathrm{C} n \mathrm{TAB}(n=10,12,14$, and 16$)$ in $20 \mathrm{mM}$ phosphate buffer of $\mathrm{pH} 7$ at $25{ }^{\circ} \mathrm{C}$.

of free or monomerically attached $\mathrm{C} n \mathrm{TAB}$ in the $\mathrm{BSA}-\mathrm{C} n \mathrm{TAB}$ complexes are different for different hydrophobic tail-length ionic surfactants because of their dissimilar hydrophobic interaction strength with the protein. Below CAC, the ionic surfactants either remain free or molecularly attached to BSA, and the overall size of the complexes is marginally increased. At CAC, a sharp rise in the hydrodynamic size of the BSA$\mathrm{C} n \mathrm{TAB}$ complexes is observed, which indicates the micelle formation. The hydrodynamic size of $\mathrm{BSA}-\mathrm{CnTAB}$ stayed almost constant with further increasing the surfactant concentration. Interestingly, a drop in the critical micelle concentration $(\mathrm{cmc})$ is observed for each surfactant, which is more prominent for the shorter tail-length cationic surfactants. The CAC is obtained as $1.0,1.5,5.5$, and $40 \mathrm{mM}$ for C16TAB, $\mathrm{C} 14 \mathrm{TAB}, \mathrm{C} 12 \mathrm{TAB}$, and $\mathrm{C} 10 \mathrm{TAB}$, respectively ( $\mathrm{cmc}$ for $\mathrm{C} 16 \mathrm{TAB}, \mathrm{C} 14 \mathrm{TAB}, \mathrm{C} 12 \mathrm{TAB}$ and $\mathrm{C} 10 \mathrm{TAB}$ are 1.0, 3.5, 15.0, and $61.8 \mathrm{mM}$, respectively). ${ }^{29}$ The decreased CAC compared 

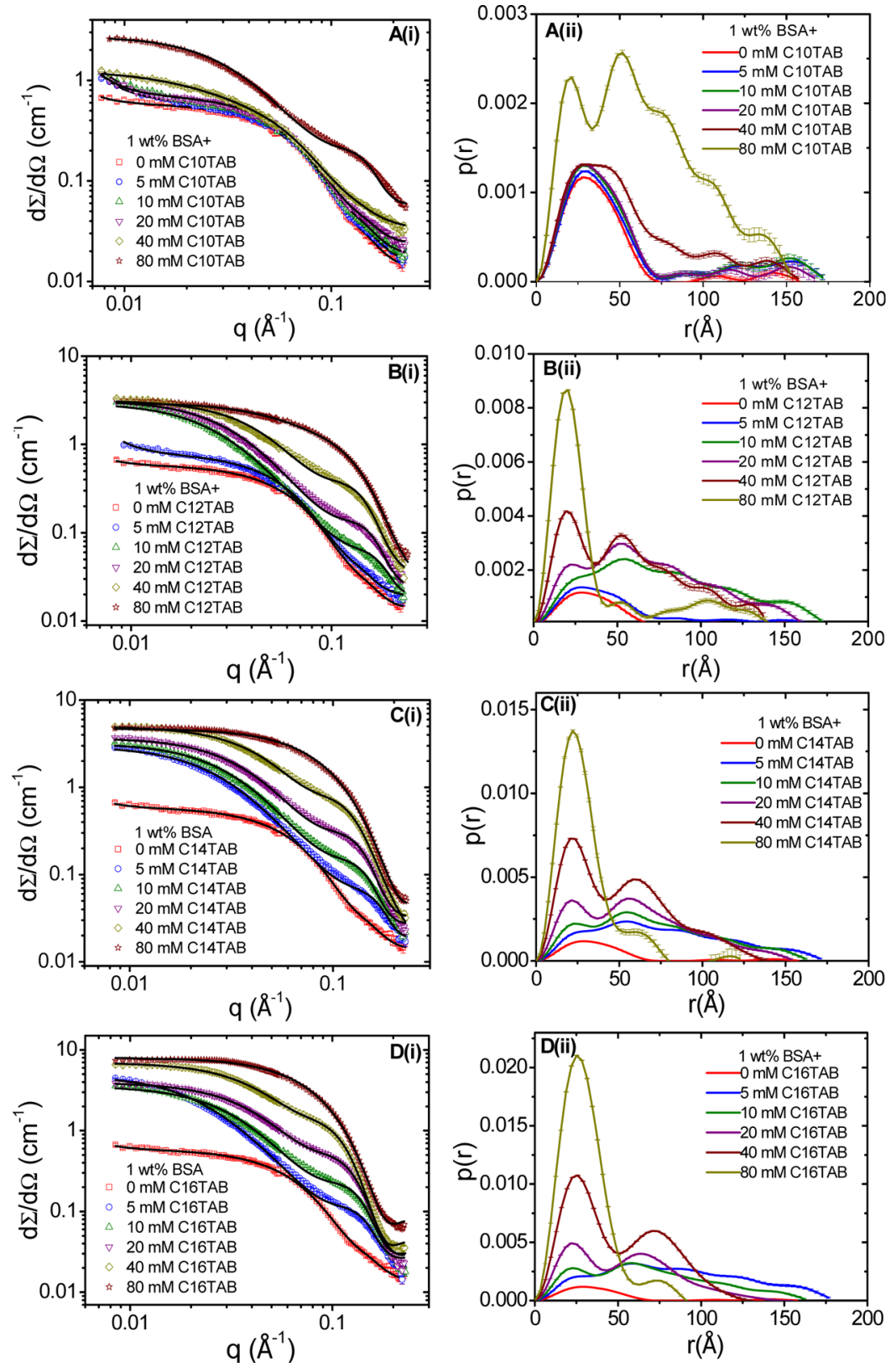

Figure 2. (Left) SANS profiles of $1 \mathrm{wt} \% \mathrm{BSA}$ in the presence of $0-80 \mathrm{mM}$ (A) C10TAB, (B) C12TAB, (C) C14TAB, and (D) C16TAB. (Right) Pair distance distribution functions $p(r)$ obtained from the fits of the experimental data.

to $\mathrm{cmc}$ results from the interaction of anionic BSA with adsorbed oppositely charged surfactants, which act as a driving force to increase the hydrophobicity of the BSA-C $n \mathrm{TAB}$ complexes. $^{15,20}$

SANS measurement is carried out for pure BSA and along with ionic surfactants having different tail lengths and at varying concentrations. The data are presented in Figure 2. No interparticle interaction (i.e., $S(q) \approx 1$ ) is observed in the scattering data of 1 wt \% BSA protein; hence, the scattering data fitted with the form factor alone. The analysis of SANS data yields an oblate ellipsoid $(\varepsilon<1)$ having semimajor axes $39.7 \AA$ and semiminor axes $14.6 \AA$, which are in good agreement with the published result. ${ }^{30}$ SANS data from individual components of $1 \mathrm{wt} \%$ ionic surfactants (C10TAB, $\mathrm{C} 12 \mathrm{TAB}, \mathrm{C} 14 \mathrm{TAB}$, and $\mathrm{C} 16 \mathrm{TAB})$ and nonionic $\mathrm{C} 12 \mathrm{E} 10$ are shown in Figure $S 1$ (Supporting Information). Micelles containing ionic $\mathrm{CnTAB}(n=10,12,14$, and 16$)$ are found to have the prolate ellipsoidal core-shell structure. ${ }^{31}$ The concentration of each of the surfactants is varied from 0 to 80 $\mathrm{mM}$. Because of the very high $\mathrm{CAC}\left(\mathrm{CAC}_{\mathrm{C} 10 \mathrm{TAB}}=40 \mathrm{mM}\right)$, $\mathrm{C} 10 \mathrm{TAB}$ molecules mostly remain free or weakly bound as monomers to the protein up to $c_{\mathrm{i}}=40 \mathrm{mM}$ (Figure 2A). Above $c_{\mathrm{i}}=40 \mathrm{mM}, \mathrm{C} 10 \mathrm{TAB}$ forms micelles with the BSA protein and unfolds its native structure. At $c_{\mathrm{i}}=80 \mathrm{mM}$, the indirect Fourier transformation (IFT) of SANS data shows the appearance of the second maximum, which indicates the formation of such a micelle-like cluster of the protein-surfactant complexes. The peak at lower- $r$ is related to the intramicelle scattering and revealed the size of the micelles, whereas the peak at larger- $r$ comes from the intermicellar interaction and reveals the center-to-center distance of one micelle to the next nearest micelle. $^{32,33}$ However, the complexes formed by the protein and the surfactant are more complicated and other 
Table 1. Fitted Parameters of SANS Data of Protein-Surfactant Systems (1 wt \% BSA $+c_{\mathrm{i}}$ mM CnTAB) Characterized by Random Flight Model Representing a Beads-On-a-String-Like Structure

\begin{tabular}{|c|c|c|c|c|c|c|c|c|c|c|}
\hline system & $c_{\mathrm{i}}(\mathrm{mM})^{a}$ & $D_{\max }(\AA)^{b}$ & $R(\AA)^{c}$ & $\varepsilon^{d}$ & $t(\AA)^{e}$ & $\rho_{\text {shell }}\left(\mathrm{cm}^{-2}\right)^{f} \times 10^{10}$ & $N_{\mathrm{CLU}}{ }^{g}$ & $D(\AA)^{h}$ & $N_{\text {agg }}^{i}$ & $N_{\text {prot/cluster }}^{j}$ \\
\hline \multirow[t]{4}{*}{ C12TAB } & 10 & 178 & $15.7 \pm 0.1$ & $1.36 \pm 0.06$ & $6.7 \pm 0.1$ & 4.96 & $13.9 \pm 1.5$ & $40 \pm 2$ & 63 & 32.6 \\
\hline & 20 & 162 & $15.8 \pm 0.1$ & $1.61 \pm 0.06$ & $6.7 \pm 0.1$ & 4.99 & $7.3 \pm 0.8$ & $47 \pm 2$ & 76 & 6.4 \\
\hline & 40 & 146 & $15.9 \pm 0.1$ & $1.71 \pm 0.07$ & $6.6 \pm 0.1$ & 5.01 & $3.0 \pm 0.4$ & $54 \pm 3$ & 82 & 1.2 \\
\hline & 80 & 68 & $15.9 \pm 0.1$ & $1.79 \pm 0.06$ & $6.6 \pm 0.1$ & 5.04 & $1.3 \pm 0.1$ & & 85 & 1.1 \\
\hline \multirow[t]{5}{*}{ C14TAB } & 5 & 179 & $17.7 \pm 0.1$ & $1.20 \pm 0.06$ & $7.3 \pm 0.1$ & 4.63 & $12.9 \pm 1.4$ & $44 \pm 3$ & 69 & 42.7 \\
\hline & 10 & 167 & $18.0 \pm 0.1$ & $1.39 \pm 0.05$ & $7.0 \pm 0.1$ & 4.79 & $6.9 \pm 0.9$ & $49 \pm 3$ & 85 & 11.4 \\
\hline & 20 & 161 & $18.2 \pm 0.2$ & $1.58 \pm 0.06$ & $6.8 \pm 0.1$ & 4.84 & $4.2 \pm 0.5$ & $53 \pm 3$ & 98 & 3.7 \\
\hline & 40 & 138 & $18.3 \pm 0.2$ & $1.74 \pm 0.06$ & $6.7 \pm 0.1$ & 4.90 & $2.2 \pm 0.2$ & $60 \pm 4$ & 110 & 1.2 \\
\hline & 80 & 81 & $18.6 \pm 0.2$ & $1.82 \pm 0.05$ & $6.4 \pm 0.1$ & 5.11 & $1.1 \pm 0.1$ & & 122 & 1.1 \\
\hline \multirow[t]{5}{*}{ C16TAB } & 5 & 190 & $19.3 \pm 0.2$ & $1.18 \pm 0.05$ & $8.3 \pm 0.1$ & 4.03 & $12.9 \pm 1.5$ & $50 \pm 3$ & 77 & 41.7 \\
\hline & 10 & 171 & $19.6 \pm 0.2$ & $1.27 \pm 0.05$ & $7.9 \pm 0.1$ & 4.14 & $5.4 \pm 0.5$ & $55 \pm 3$ & 88 & 8.8 \\
\hline & 20 & 147 & $19.9 \pm 0.2$ & $1.43 \pm 0.04$ & $7.6 \pm 0.1$ & 4.26 & $2.7 \pm 0.2$ & $60 \pm 3$ & 103 & 2.4 \\
\hline & 40 & 129 & $21.4 \pm 0.3$ & $1.56 \pm 0.04$ & $6.2 \pm 0.1$ & 5.08 & $1.9 \pm 0.1$ & $67 \pm 4$ & 140 & 1.1 \\
\hline & 80 & 91 & $21.8 \pm 0.3$ & $1.66 \pm 0.05$ & $5.8 \pm 0.1$ & 5.35 & $1.1 \pm 0.1$ & & 157 & 1.1 \\
\hline
\end{tabular}

${ }^{a}$ Ionic surfactant concentration in $\mathrm{mM} .{ }^{b}$ Maximum dimension of the protein-C $n \mathrm{TAB}(n=12,14$, and 16$)$ complexes derived from the $p(r)$ function obtained by IFT. ${ }^{c}$ Semiminor axis of the micellar core. ${ }^{d}$ Axial anisotropy in the size of micelles. ${ }^{e}$ Thickness of the shell of the micelle. ${ }^{f}$ Contrast of the micellar shell. ${ }^{g}$ Number of micelles per cluster. ${ }^{h}$ Separation between the center of two nearest micelles. ${ }^{i}$ Aggregation number of micelles in terms of the number of $\mathrm{C} n \mathrm{TAB}$ molecules that make up each micelle. ${ }^{j}$ Number of proteins per cluster, given as the number of micelles per cluster multiplied by the number of proteins per micelle.

intermicellar correlations may come, which is hard to model through the $p(r)$ function.

The tail-length-dependent structural changes of the BSA$\mathrm{C} n \mathrm{TAB}(n=12,14$, and 16$)$ complexes can be explained from the shape of $p(r)$. The formation of the micelle-like clusters in the protein-surfactant complexes for all the surfactants is indicated by the appearance of multidomain aggregates. The similarity of the $p(r)$ function of $1 \mathrm{wt} \% \mathrm{BSA}+5 \mathrm{mM} \mathrm{C12TAB}$ complexes with $1 \mathrm{wt} \% \mathrm{BSA}$ demonstrates that $5 \mathrm{mM} \mathrm{C12TAB}$ is unable to perturb the native structure of BSA because of insufficient hydrophobic force to form micelles. The appearance of the second maximum of 1 wt $\%$ BSA +10 $\mathrm{mM}$ C12TAB complexes suggests the micelle-like cluster formation. This length scale as already mentioned is related to the correlation between two nearest micelles in the cluster. Another interesting feature is obtained from $p(r)$ that the amplitude of oscillation at the first bump increased with increasing surfactant concentration, which reflects the occurrence of a better-defined micelle structure or an increased concentration of free micelles in solution. In addition, the cluster size decreases systematically with increasing C12TAB concentration (see the values of $D_{\max }$ in Table 1 ). Thus, a higher surfactant to protein ratio enables the formation of the smaller cluster. A similar trend is observed in the $p(r)$ function of $\mathrm{BSA}-\mathrm{C} 14 \mathrm{TAB}$ and $\mathrm{BSA}-\mathrm{C} 16 \mathrm{TAB}$ complexes. However, the second maximum appears in the $p(r)$ even at the lowest added surfactant concentration $\left(c_{\mathrm{i}}=5 \mathrm{mM}\right)$ with $\mathrm{C} 14 \mathrm{TAB}$ and C16TAB. This is a clear indication of the micelle formation of 1 wt $\%$ BSA +5 mM CnTAB $(n=14$ and 16$)$ complexes. At $c_{\mathrm{i}}$ $=80 \mathrm{mM}$, the first maximum is raised to the highest amplitude, whereas the second maximum is almost disappeared in the $p(r)$ function of BSA-C $n \mathrm{TAB}(n=12,14$, and 16$)$ complexes. The disappearance of the second maximum suggests the change in the morphology of the cluster. IFT fit of SANS data are presented in Figure S2 (Supporting Information).

The detailed modeling of SANS data obtained from BSA$\mathrm{CnTAB}$ complexes at different concentrations is shown in Figure 2 (left). It is observed that the scattering profiles of $\mathrm{BSA}-\mathrm{C} 10 \mathrm{TAB}$ complexes at the concentrations ranging from
5 to $40 \mathrm{mM}$ have behavior similar to that of pure protein and confirmed by $p(r)$ measurement of the scattering intensity (Figure $2 \mathrm{~A}(\mathrm{ii})$ ). The SANS profiles of BSA-C10TAB clearly suggest the absence of the micellar structure in the sub-CAC concentration range of $\mathrm{C} 10 \mathrm{TAB}$, and therefore, the native structure of $\mathrm{BSA}$ remains unperturbed. The interparticle interaction is clearly visible in the SANS data on further increasing the C10TAB concentration $\left(c_{\mathrm{i}}=80 \mathrm{mM}\right)$. Therefore, $S(q)$ of the micelles organized in a cluster using the random flight model along with $P(q)$ of ellipsoidal coreshell micelles is employed to extract the information from SANS data. The fitted parameters of the protein with different tail-length surfactants are given in Table 1 .

The scattering profile of BSA-C12TAB complexes shows behavior similar to that of native protein solution at $c_{\mathrm{i}}=5 \mathrm{mM}$. The scattering profiles of protein-surfactant complexes above CAC ( $5.5 \mathrm{mM})$ of C12TAB have a completely different feature to those below CAC. At $c_{\mathrm{i}}=10 \mathrm{mM}$, the linearity of the scattering profile in the intermediate- $q$ range is increased, and a strong change in the scattering profile in the low- $q$ range is also observed. The fitting of SANS data by a random flight model as described in eq 4 yields that as the number of $\mathrm{C} 12 \mathrm{TAB}$ molecules is increased, the micelles get elongated in one direction and the axial ratio $(\varepsilon)$ is also increased. On the other hand, the thickness of the shell is decreased while the scattering length density of the shell increased at higher $\mathrm{C} 12 \mathrm{TAB}$ concentration. With increasing the C12TAB concentration from 10 to $80 \mathrm{mM}$, the number of micelles per cluster $\left(N_{\mathrm{CLU}}\right)$ is decreased from 13.5 to 1.2 , clearly suggesting that the cluster is replaced by a single elongated micelle. Following a similar trend, the number of proteins per cluster is also decreased and finally reduced to $\sim 1$ at a higher $\mathrm{C} 12 \mathrm{TAB}$ concentration. By contrast, the distance between the micelles is increased from 41 to $64 \AA$ (see Table 1 ). At the beginning of the unfolding process, micelles with an aggregation number of $63 \mathrm{C} 12 \mathrm{TAB}$ molecules are formed, which jumped to 85 to stabilize the elongated micelle at the final stage of the unfolding process. The present analysis suggests that the beads-on-a-string-type structure disappeared 

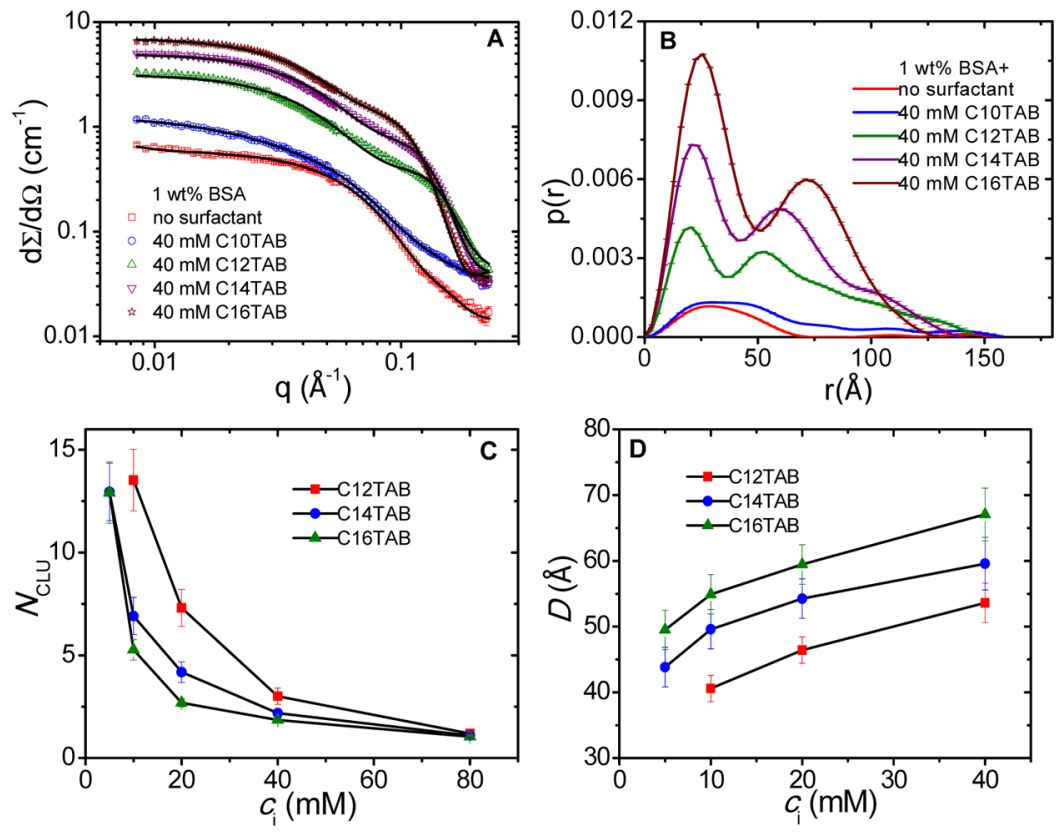

Figure 3. (A) SANS profile of 1 wt $\%$ BSA with $40 \mathrm{mM}$ of C10TAB, C12TAB, C14TAB, and C16TAB. (B) Pair distance distribution function $p(r)$ obtained from the fits of the experimental data. (C) Number of micelles per cluster for $1 \mathrm{wt} \% \mathrm{BSA}+40 \mathrm{mM} \mathrm{C} n \mathrm{TAB}(n=12,14$, and 16$)$ complexes and (D) distance between two nearest micelles in the micelle-like cluster.

at a higher $\mathrm{C} 12 \mathrm{TAB}$ concentration, and the protein molecules go through a structural redistribution and wrap the single micelle through the surface.

SANS data of 1 wt $\%$ BSA protein with increasing concentration of $\mathrm{C} 14 \mathrm{TAB}$ and $\mathrm{C} 16 \mathrm{TAB}$ ranging from 0 to $80 \mathrm{mM}$ are shown in Figure 2C,D, respectively. The scattering features of the BSA-C $n \mathrm{TAB}(n=14$ and 16) system at different concentrations significantly differ from each other. Because of the very low CAC of C14TAB and C16TAB, they have displayed strong hydrophobic interaction with BSA through micelle formation. The concentration window, in which the protein-surfactant interaction is totally electrostatic, is not observed for $\mathrm{BSA}-\mathrm{C} n \mathrm{TAB}(n=14$ and 16) complexes in the measured concentration range. In fact, at the lowest added surfactant concentration $(5 \mathrm{mM})$, the scattering profile is already significantly different from the native BSA structure. This suggests that the denaturation of BSA starts at the lowest added concentration of $\mathrm{C} 14 \mathrm{TAB}$ and $\mathrm{C} 16 \mathrm{TAB}\left(c_{\mathrm{i}}=5 \mathrm{mM}\right)$. The analysis depicts that the micelle size, micellar anisotropy $(\varepsilon)$, and the aggregation number increased with increasing the surfactant concentration and thus enhance the denaturation of BSA. In addition to that, the shell thickness is decreased while the scattering length density of the shell increased at higher $\mathrm{C} 14 \mathrm{TAB}$ and $\mathrm{C} 16 \mathrm{TAB}$ concentration. On the other hand, the number of micelles per cluster $\left(N_{\mathrm{CLU}}\right)$ and the number of proteins per cluster are decreased, whereas the distance $D$ increased with increasing the surfactant concentration. Similar to the $\mathrm{BSA}-\mathrm{C} 12 \mathrm{TAB}$ system, at the highest added concentration $\left(c_{\mathrm{i}}=80 \mathrm{mM}\right)$ of $\mathrm{C} 14 \mathrm{TAB}$ and $\mathrm{C} 16 \mathrm{TAB}$, the analysis yields the merging of smaller micelles in the cluster to a single elongated micelle $\left(N_{\mathrm{CLU}}=1\right)$.

A very significant trend revealed from this study is that with increasing the tail length of the ionic surfactant, the hydrophobic interaction of $\mathrm{BSA}-\mathrm{Cn} \mathrm{TAB}$ complexes gets stronger, and thus the protein is unfolded at lower concentrations of surfactants. To draw a more detailed picture of the hydrophobic tail-length effect in the protein-surfactant interaction, the SANS profile of 1 wt $\%$ BSA with $40 \mathrm{mM}$ surfactants is compared in Figure 3. The scattering features of BSA with different tail-length ionic surfactants are significantly different from each other. The $p(r)$ function of 1 wt \% BSA + $40 \mathrm{mM}$ C10TAB complexes has a behavior similar to $1 \mathrm{wt} \%$ BSA. The second maximum which is related to the micelle-like cluster formation is clearly missing in this system. The SANS data of BSA with $40 \mathrm{mM} \mathrm{C10TAB}$ are interpreted as a sitespecific electrostatic binding of monomeric surfactant molecules on the oppositely charged patches of BSA. It is observed that with increasing the hydrophobic tail length, the amplitude of the first oscillation is increased and its position is shifted to larger- $r$ in the $p(r)$ function (Figure 3B). This is an indication of the bigger micelle structure formation by BSA with higher tail-length surfactants. The increase in the micelle size is consistent with the position of high- $q$ cutoff, which is shifted to lower- $q$ value with increasing the surfactant tail length. The appearance of the second maximum at higher tail-length surfactants corresponds to the micelles-decorated protein clusters. The number of micelles per cluster and the distance between the micelles are compared to see the propensity of protein unfolding with increasing hydrophobic tail-length of the ionic surfactant (Figure 3C,D). At $c_{\mathrm{i}}=40 \mathrm{mM}$, the biggest but least micelles are obtained for BSA-C16TAB complexes (Table 1). These results suggest the higher propensity of the unfolding of C16TAB compared to other surfactants. As the number of micelles per cluster is decreased more rapidly, the distance between two micelles gets larger for BSA $+40 \mathrm{mM}$ $\mathrm{C} 16 \mathrm{TAB}$ complexes compared to others (Figure 3D). The higher aggregation number of $\mathrm{C} 16 \mathrm{TAB}$ in the proteinsurfactant complexes is also responsible for the higher propensity of unfolding by this surfactant. On the contrary, the higher number of micelles with low aggregation number suggests the weaker denaturation of BSA in the BSA-C12TAB complexes compared to other systems having longer tail-length surfactants. These results thus show that the hydrophobic 

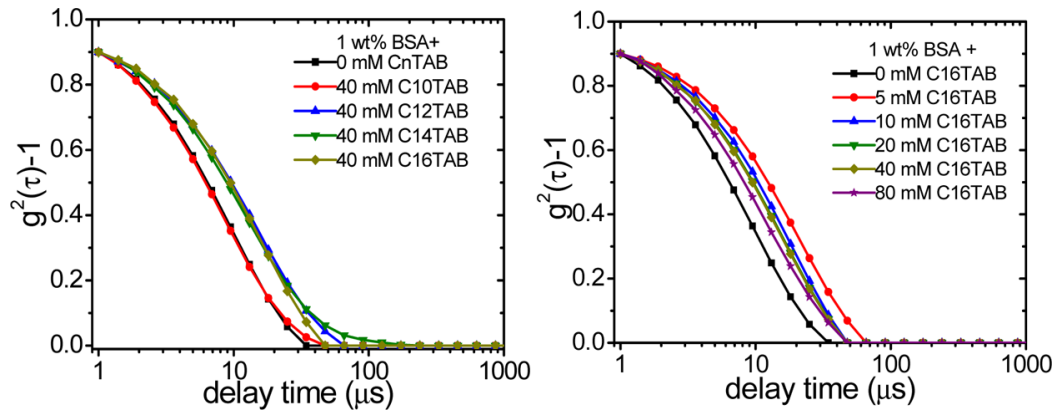

Figure 4. DLS data of protein-surfactant complexes of (left) $1 \mathrm{wt} \% \mathrm{BSA}+40 \mathrm{mM} \mathrm{CnTAB}(n=10,12,14$, and 16$)$ and (right) $1 \mathrm{wt} \% \mathrm{BSA}+c_{\mathrm{i}}$ $\mathrm{mM} \mathrm{C} 16 \mathrm{TAB}\left(c_{\mathrm{i}}=5-80 \mathrm{mM}\right)$.
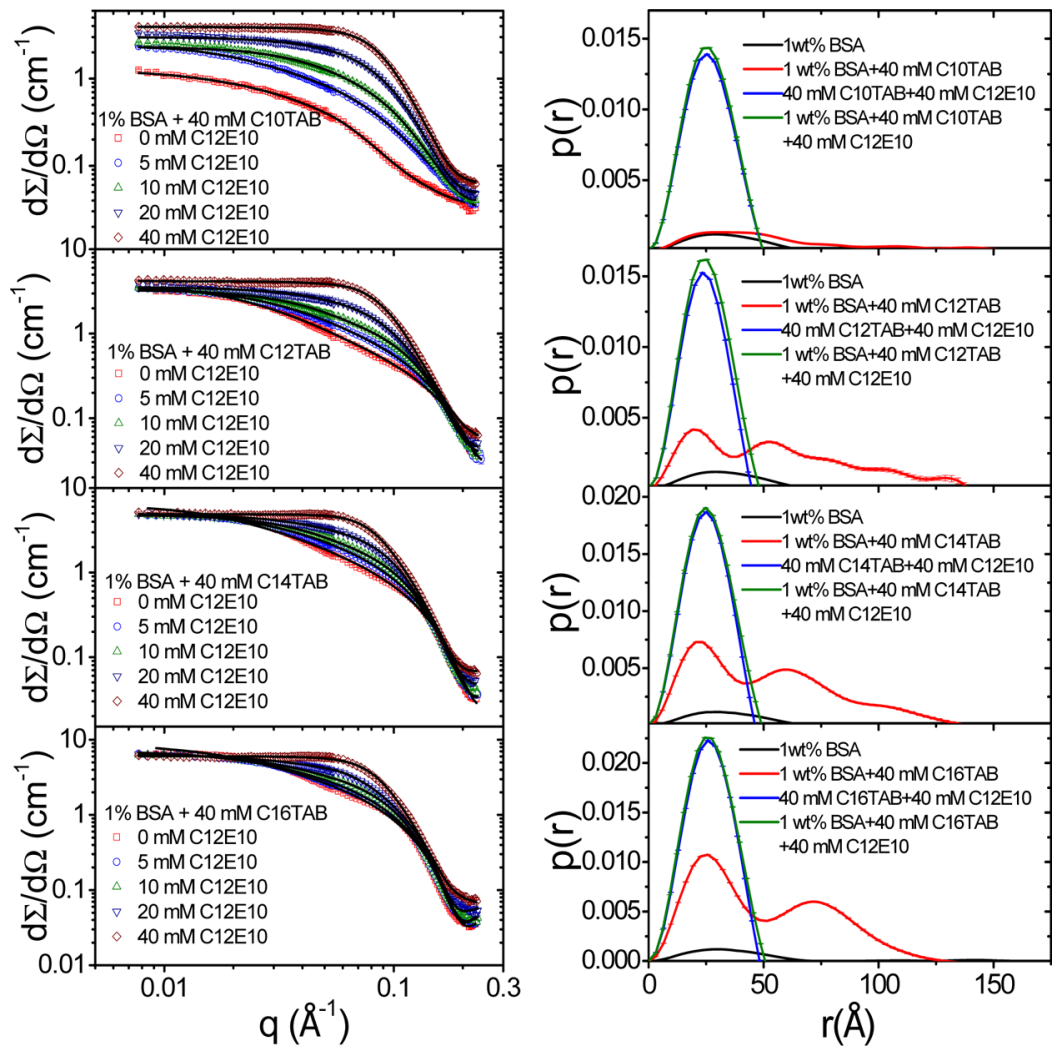

Figure 5. (Left) SANS data of 1 wt $\%$ BSA with $40 \mathrm{mM} \mathrm{C} n \mathrm{TAB}(n=10,12,14$, and 16$)$ with varying concentrations of C12E10 and (right) pair distance distribution functions $p(r)$ obtained from the fits of the experimental data of native BSA, BSA with $40 \mathrm{mM} \mathrm{C} n \mathrm{TAB}(n=10,12,14$, and $16)$, ionic-nonionic mixed micelles, and BSA with ionic-nonionic mixed micelles.

interaction between the protein and surfactants is enhanced with increasing the hydrophobic tail length of ionic surfactants.

DLS is used to support the conclusions from SANS for the BSA-CnTAB complexes. The differences in the decay of the intensity autocorrelation function $g^{2}(\tau)$ are related to the different diffusion coefficients of these systems. ${ }^{34}$ DLS data of 1 wt $\%$ BSA-CnTAB complexes at $c_{\mathrm{i}}=40 \mathrm{mM}$ and 1 wt $\%$ BSA with $c_{\mathrm{i}} \mathrm{C} 16 \mathrm{TAB}\left(c_{\mathrm{i}}=5-80 \mathrm{mM}\right)$ are presented in Figure 4. The decay of the intensity autocorrelation function of the native protein is faster compared to $\mathrm{BSA}-\mathrm{C} n \mathrm{TAB}$ complexes except for $n=10$. The superposition of intensity autocorrelation function of $\mathrm{BSA}-\mathrm{C} 10 \mathrm{TAB}$ complexes with the native protein indicates that no unfolding of BSA takes place in the presence of $40 \mathrm{mM} \mathrm{C10TAB}$, which is also observed from SANS. On the contrary, the slower decay of the intensity correlation function of $\mathrm{BSA}-\mathrm{C} n \mathrm{TAB}$ complexes (except $n=10$ ) indicates micelles' formation because of the strong hydrophobic interaction of the protein and surfactants. 935 The hydrodynamic radii are calculated using the Stokes-Einstein relation presented in eq 5 (Table S1, Supporting Information). The increase in the hydrodynamic size of protein-surfactant complexes supports the unfolding of BSA being determined by the protein-surfactant hydrophobic interaction. The hydrodynamic sizes obtained from the intensity autocorrelation can be compared to the maximum dimension of the BSA-CnTAB complex derived from the $p(r)$ function obtained by IFT. The trend observed from DLS is in good agreement with the results obtained from SANS analysis for the structural evolution of protein-surfactant complexes with varying concentrations and tail lengths of the surfactant. DLS data of $1 \mathrm{wt} \% \mathrm{BSA}+c_{\mathrm{i}} \mathrm{C} 16 \mathrm{TAB}$ yields that the size of protein-surfactant complexes is decreased at higher surfactant concentrations $\left(c_{\mathrm{i}}>10 \mathrm{mM}\right)$. SANS analysis shows that with increasing surfactant concentration, lesser micelles are formed, 

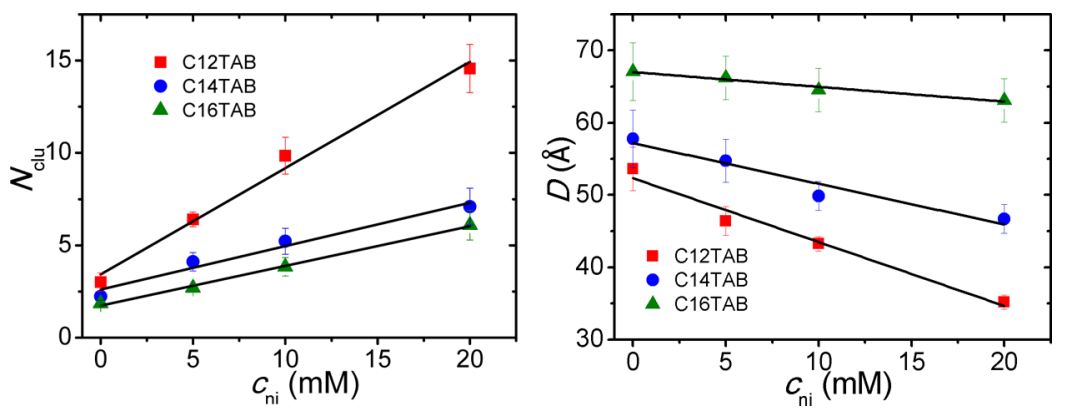

Figure 6. (Left) Number of micelles per cluster and (right) distance between micelles in the cluster for BSA $+40 \mathrm{mM} \mathrm{CnTAB}(n=12,14$, and 16$)$ $+c_{\mathrm{ni}} \mathrm{mM} \mathrm{C12 \textrm {E } 1 0}\left(c_{\mathrm{ni}}=0,5,10\right.$, and 20$)$ complexes.

which might be the reason for smaller sizes in DLS at higher surfactant concentrations. A similar trend is also observed in the $p(r)$ function at higher surfactant concentrations (Figure $2)$. The DLS results of BSA-CnTAB $(n=10,12$, and 14) complexes at different surfactant concentrations are presented in Figure S3 (Supporting Information).

2.2. Effect of Mixed Surfactants: Refolding of BSA

Protein. It is observed that the interactions of BSA with ionic and nonionic surfactants are dissimilar from each other. The presence of ionic surfactants in the protein-surfactant complexes electrostatically and hydrophobically interact with proteins and denature its native structure. On the contrary, the nonionic surfactants do not interact with the protein, whereas they form mixed micelles with ionic surfactants through the hydrophobic interaction. In a recent study, it is shown that the presence of nonionic $\mathrm{C} 12 \mathrm{E} 10$ in the BSA-C12TAB complexes reinforces the refolding of BSA. ${ }^{11}$ In another study, the pathway of nonionic surfactant-dependent refolding of protein unfolded by the ionic surfactant has been introduced. ${ }^{13}$ In the present study, it is demonstrated that in the protein-ionic surfactant complexes, the propensity of the unfolding of protein can be modified through the hydrophobic interaction by changing the hydrophobic tail length of the surfactant. The role of a nonionic surfactant for refolding of unfolded protein with different tail-length ionic surfactants is further examined. For this purpose, ternary systems consisting of BSA, nonionic surfactant, C12E10, and a series of ionic surfactants with different tail lengths are investigated.

The SANS profiles of 1 wt $\%$ BSA $+40 \mathrm{mM} \mathrm{CnTAB}(n=10$, 12,14 , and 16) in the presence of C12E10 with different concentrations are presented in Figure 5. The figure also shows $p(r)$ of some of the selected concentrations of C12E10. It is already shown that the presence of $40 \mathrm{mM} \mathrm{C10TAB}$ is unable to denature the native structure of $\mathrm{BSA}$. Therefore, the scattering features of BSA-C10TAB complexes with C12E10 are defined by the sum of the scattering contributions from pure protein and ionic-nonionic surfactant mixed micelles. The argument is supported by the absence of the second maximum in the $p(r)$ function of $1 \mathrm{wt} \% \mathrm{BSA}+40 \mathrm{mM}$ $\mathrm{C} 10 \mathrm{TAB}+c_{\mathrm{ni}} \mathrm{C} 12 \mathrm{E} 10\left(c_{\mathrm{ni}}=5-40 \mathrm{mM}\right)$ complexes. On the contrary, the scattering features obtained for BSA with $40 \mathrm{mM}$ $\mathrm{CnTAB}(n=12,14$, and 16$)$ and $5 \mathrm{mM} \mathrm{C12E} 10$ are different from the 1 wt $\%$ BSA $+40 \mathrm{mM} \mathrm{C10TAB}+5 \mathrm{mM} \mathrm{C} 12 \mathrm{E} 10$ system but similar to those of protein-ionic surfactant systems. Above $5 \mathrm{mM}$ concentration of C12E10, the scattering features of protein with mixed surfactants are very different from the unfolded protein-ionic surfactants complexes. Four possible scenarios are considered for the interaction of BSA with the mixed surfactant system. First, it is assumed that only the ionic surfactant interacts with BSA, which leads to the formation of the ionic surfactant-induced unfolded BSA that coexists with noninteracting nonionic micelles (model 1). Second, both ionic and nonionic surfactants interact with the protein and yield mixed surfactant-induced protein unfolding (model 2). Third, the ionic surfactant-induced unfolded protein coexists with the ionic-nonionic mixed surfactant micelles (model 3) and finally, none of the surfactants interact with the protein and the scattering profile becomes a sum of scattering contributions from native BSA and mixed surfactants (model 4). The simulated data of these four scenarios are presented in Figure S4 (Supporting Information).

At a lower concentration of C12E10 $\left(c_{\mathrm{ni}}=5 \mathrm{mM}\right)$, the analysis of SANS data and the calculated $p(r)$ function show less BSA unfolding for $1 \mathrm{wt} \% \mathrm{BSA}+40 \mathrm{mM} \mathrm{C} n \mathrm{TAB}(n=12$,

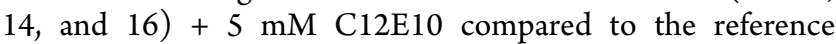
complexes $[1$ wt $\% \mathrm{BSA}+40 \mathrm{mM} \mathrm{CnTAB}(n=12,14$, and 16)]. A fraction of ionic surfactants is extracted out from the protein-surfactant complexes because of the hydrophobic interaction between the ionic and nonionic surfactants, which reduces the propensity of unfolding. Therefore, the coexistence of mixed micelles with unfolded protein (model 3) gives good agreement between the measured scattering and the constructed model. The hydrophobic interaction increases with increasing the concentration of C12E10 in the proteinsurfactant complexes. The stronger hydrophobic force extracts more and more ionic surfactants and results in the increasing of the number of micelles in the cluster, whereas the size and the spacing between the micelles decrease significantly (Figure 6). This is completely reverse to the case of protein unfolding, where the number of micelles per cluster decreased and the micelle spacing increased with increasing concentration of ionic surfactants. At $c_{\mathrm{ni}}=40 \mathrm{mM} \mathrm{C12E10,} \mathrm{the} \mathrm{scattering} \mathrm{data}$ fitted well to model 4 , where the refolded BSA coexists with mixed micelles of ionic and nonionic surfactants. The second maximum in the $p(r)$ function, which is related to the formation of micelle-like complexes, disappeared in this concentration. In contrast, the huge difference between the experimental data and the constructed model rules out the possibility of noninteracting nonionic micelles' coexistence with ionic micelles-induced unfolded protein and mixed surfactant-induced protein unfolding (model 1 and model 2 ).

The second conclusion is that irrespective of the tail length of ionic surfactants, $\mathrm{C} 12 \mathrm{E} 10$ changes them from interacting to noninteracting with protein. The phenomena can be understood based on the competition of electrostatic binding of the ionic surfactant with protein with that of the hydrophobic interaction of the ionic surfactant with a nonionic surfactant. At the initial stage of C12E10 addition, the weak interaction of 
Table 2. Fitted Parameters of SANS Data of the Protein-Surfactant System (1 wt \% BSA $\left.+40 \mathrm{mM} \mathrm{CnTAB}+c_{\mathrm{ni}} \mathrm{C12E10}\right)$ Characterized by the Random Flight Model Representing a Beads-On-a-String-Like Structure

\begin{tabular}{|c|c|c|c|c|c|c|c|}
\hline system & $c_{\mathrm{ni}}(\mathrm{mM})^{a}$ & $R(\AA)^{b}$ & $\varepsilon^{c}$ & $t^{d}(\AA)$ & $\rho_{\text {shell }}^{e}$ & $N_{\mathrm{CLU}}^{f}$ & $D(\AA)^{g}$ \\
\hline \multirow[t]{4}{*}{$1 \mathrm{wt} \% \mathrm{BSA}+40 \mathrm{mM} \mathrm{C12TAB}$} & 5 & $16.4 \pm 0.1$ & $1.67 \pm 0.05$ & $6.0 \pm 0.1$ & 5.53 & $6.4 \pm 0.5$ & $48 \pm 2$ \\
\hline & 10 & $16.3 \pm 0.1$ & $1.31 \pm 0.04$ & $6.1 \pm 0.1$ & 5.50 & $9.9 \pm 1.0$ & $44 \pm 1$ \\
\hline & 20 & $16.2 \pm 0.1$ & $1.22 \pm 0.04$ & $6.3 \pm 0.1$ & 5.47 & $14.6 \pm 1.3$ & $34 \pm 1$ \\
\hline & 40 & & & & & & \\
\hline \multirow[t]{4}{*}{1 wt $\%$ BSA $+40 \mathrm{mM} \mathrm{C14TAB}$} & 5 & $18.3 \pm 0.1$ & $1.53 \pm 0.04$ & $6.7 \pm 0.1$ & 5.37 & $4.1 \pm 0.5$ & $55 \pm 3$ \\
\hline & 10 & $18.1 \pm 0.1$ & $1.38 \pm 0.05$ & $6.9 \pm 0.1$ & 5.31 & $5.2 \pm 0.7$ & $50 \pm 2$ \\
\hline & 20 & $18.0 \pm 0.1$ & $1.14 \pm 0.05$ & $7.0 \pm 0.1$ & 5.28 & $7.1 \pm 1.0$ & $46 \pm 2$ \\
\hline & 40 & & & & & & \\
\hline \multirow[t]{3}{*}{1 wt $\%$ BSA $+40 \mathrm{mM} \mathrm{C} 16 \mathrm{TAB}$} & 5 & $19.3 \pm 0.2$ & $1.76 \pm 0.05$ & $8.2 \pm 0.1$ & 4.94 & $2.7 \pm 0.2$ & $66 \pm 3$ \\
\hline & 10 & $19.1 \pm 0.2$ & $1.39 \pm 0.05$ & $8.4 \pm 0.1$ & 4.85 & $3.8 \pm 0.5$ & $64 \pm 3$ \\
\hline & 20 & $18.9 \pm 0.1$ & $1.13 \pm 0.04$ & $8.6 \pm 0.1$ & 4.75 & $6.1 \pm 0.8$ & $62 \pm 3$ \\
\hline
\end{tabular}

${ }^{a}$ Nonionic surfactant concentration in $\mathrm{mM} .{ }^{b}$ Semiminor axis of the micellar core. ${ }^{c}$ Axial anisotropy in the size of micelles. ${ }^{d}$ Shell thickness. ${ }^{e}$ Scattering length density of the shell. ${ }^{f}$ Number of micelles per cluster. ${ }^{g}$ Separation between the center of two nearest micelles.

ionic and nonionic surfactant is unable to overcome the electrostatic binding of BSA-ionic surfactants. However, a significant fraction of ionic surfactants is extracted from the protein-surfactant complexes. With increasing C12E10 concentration, the hydrophobic interaction between ionic and nonionic surfactants increased and above a certain concentration of the nonionic surfactant, the hydrophobic interaction gets total control over the electrostatic binding of ionic surfactants with protein. As a result of the dominance of hydrophobic interaction over the electrostatic one, BSA regained its native structure.

These scenarios are modeled through the increasing number of micelles per cluster, decreasing the separation between micelles and anisotropy in the micelle structure (Figure 6). It is noteworthy that as the interaction between the ionic and nonionic micelles gets stronger, the micellar shell thickness is also increased. In contrast, the scattering length density of the shell is decreased. It is observed that the pathway of refolding of BSA consisting of different cationic surfactants is more or less the same. However, the propensity of refolding significantly decreases with the increase in the tail length of the ionic surfactant. C16TAB shows the lowest refolding efficiency by combining with $\mathrm{C} 12 \mathrm{E} 10$ compared to other ionic surfactants because of its stronger interaction with BSA protein. The analyses of SANS data of 1 wt $\%$ BSA +40 $\operatorname{mMC} n \operatorname{TAB}(n=12,14$, and 16$)+c_{\mathrm{ni}} \operatorname{mM~C12E10~}\left(c_{\mathrm{ni}}=5\right.$, 10,20 , and 40) are given in Table 2.

The refolding of unfolded BSA protein in the presence of nonionic C12E10 is further confirmed by DLS measurements. Figure 7 shows the variation in the intensity autocorrelation function with increasing concentration of C12E10 in the BSA$\mathrm{C} 16 \mathrm{TAB}$ complexes. The intensity autocorrelation function decays faster with increasing concentration of C12E10. At $c_{\mathrm{ni}} \approx$ $120 \mathrm{mM}$ of C12E10, the refolding of BSA is completed and the intensity autocorrelation function exactly overlapped with the autocorrelation function of the native BSA one. This suggests the coexistence of refolded BSA with ionic and nonionic surfactant mixed micelles in the system. The hydrodynamic diameter of protein-surfactant complexes with increasing C12E10 concentration is presented in the inset. The hydrodynamic size decreases with $\mathrm{C} 12 \mathrm{E} 10$ up to $120 \mathrm{mM}$ and thereafter remains constant and equal to that of the folded protein. The effect of the addition of C12E10 to BSA$\mathrm{C} 12 \mathrm{TAB}$ and $\mathrm{BSA}-\mathrm{C} 14 \mathrm{TAB}$ complexes is also found similar

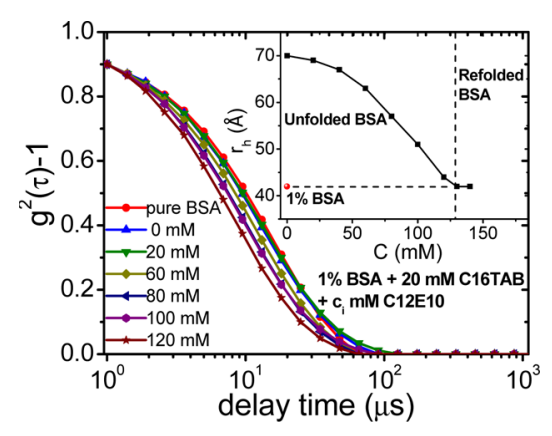

Figure 7. DLS data of $1 \mathrm{wt} \% \mathrm{BSA}$ and $20 \mathrm{mM} \mathrm{C} 16 \mathrm{TAB}$ with varying concentrations of C12E10. The inset shows the variation of the hydrodynamic radius with the change in the concentration of C12E10.

to that of BSA-C16TAB (Figure S5, Supporting Information). It is to be mentioned that the size of the ionic-nonionic surfactants' mixed micelles is marginally smaller than the size of the native protein (Figure S6, Supporting Information). Therefore, it is really difficult to extract the contributions of individual components by DLS.

The present study demonstrates the nonionic surfactantdependent refolding of ionic surfactant-induced unfolded protein through mixed micelles formation. The combined study of SANS and DLS in BSA protein unfolding and refolding experiments allows us to probe the overall features of the protein. In general, the transitions from folding to unfolding and back to refolding are reversible and easy to follow. The ability to refold BSA at a low concentration of C12E10 to form micelle-free structures demonstrates the advantage of transferring $\mathrm{C} n \mathrm{TAB}(n=10,12,14$, and 16) molecules into mixed micelles with a very favorable mixing entropy. This clearly indicates that BSA proteins are not kinetically trapped in the unfolded state. The redistribution of cationic surfactants into mixed micelles with the nonionic surfactant makes it possible to withdraw sufficient amounts of $\mathrm{C} n \mathrm{TAB}(n=10,12,14$, and 16$)$ from the complexes to allow the proteins to refold back.

\section{CONCLUSIONS}

The emerging perspective from the current study is that the unfolding and refolding of BSA protein can be controlled by the hydrophobic interaction between protein and surfactants 
via changing the hydrophobic tail length of the ionic surfactant. The anionic protein interacts differently with cationic and nonionic surfactants. The ionic surfactant binds to the protein by site-specific electrostatic interaction and forms beads-on-astring chain-like complexes along the unfolded peptide chain of protein. With increasing hydrophobic tail length of the ionic surfactant, the BSA protein unfolds even at lower concentrations through the micelle formation, which reveals that the propensity of BSA unfolding increases with increasing the hydrophobic tail length of the ionic surfactant. It is noteworthy that the addition of a nonionic surfactant results in folding back of the unfolded protein. The interplay of the hydrophobic interaction of mixed surfactants with the electrostatic interaction of the ionic surfactant and the protein determines the pathway of the refolding process. It is easier to overcome the interaction of BSA with shorter tail length ionic surfactants by adding nonionic C12E10 because of their weaker interaction. This implies that the propensity of refolding decreases with increasing the hydrophobic tail length of the ionic surfactant. The present study thus shows that it is possible to regulate the unfolding and refolding of BSA by changing the hydrophobic tail length of the ionic surfactant. The idea remains to be consolidated by more extensive future studies with a series of nonionic surfactants with different carbon chains as well as multimeric proteins.

\section{EXPERIMENTAL SECTION}

4.1. Materials. BSA with $99 \%$ purity was purchased from Sigma-Aldrich and used without any purification. The received protein was a lyophilized powder with the molecular weight of $\sim 66 \mathrm{kDa}$. Cationic C10TAB, C12TAB, C14TAB, C16TAB, and nonionic C12E10 were purchased from Sigma-Aldrich. Samples were prepared in $\mathrm{D}_{2} \mathrm{O}$ (99.9 atom \% D) to ensure sufficient contrast for SANS experiments, ${ }^{36}$ whereas they were prepared in $\mathrm{H}_{2} \mathrm{O}$ for DLS measurements. In the first step, the individual components (BSA and ionic and nonionic surfactants) were dissolved in $20 \mathrm{mM}$ phosphate buffer at $\mathrm{pH}=7.0$. They were kept at room temperature overnight to allow them to mix properly. For the unfolding study, the measurements were carried out for a fixed concentration of protein $(1 \mathrm{wt} \%)$ and varying concentrations of surfactants $\left(c_{\mathrm{i}}\right)$ in the range of $0-80 \mathrm{mM}$. The tail length of $\mathrm{CnTAB}$ was varied from $n=10$ to 16 . For the refolding study, the C12E10 concentration $\left(c_{\mathrm{ni}}\right)$ was varied from 0 to $40 \mathrm{mM}$ while keeping the BSA and ionic surfactant contents fixed. In the first step of sample preparation, the stock solution of the ionic surfactant was mixed with the protein solution to denature the protein. In the next step, the stock solution of the nonionic surfactant was added to the protein-ionic surfactant complexes to refold back the unfolded protein.

4.2. Small-Angle Neutron Scattering. SANS experiments were carried out on the SANS-I facility at the Swiss Spallation Neutron Source SINQ Paul Scherrer Institute, Switzerland. ${ }^{37}$ All the measurements were performed at $\lambda=8$ $\AA$ and the wavelength resolution $(\Delta \lambda / \lambda)$ was about $10 \%$. Samples were put in a $2 \mathrm{~mm}$ HELLMA quartz cuvette and aligned using the thermostatic sample holder with a fixed temperature of $25{ }^{\circ} \mathrm{C}$. A two-dimensional $\left(96 \times 96 \mathrm{~cm}^{2}\right){ }^{3} \mathrm{He}$ gas detector was used to detect the scattered neutrons. Two sample-to-detector distances of 2 and $8 \mathrm{~m}$ were used to cover the $q$-range from 0.007 to $0.30 \AA^{-1}$. The raw data were radially averaged, corrected for electronic background and empty cell, and normalized by water scattering using the BerSANS software. $^{38}$

In the first step of data treatment, an IFT is applied to the scattering data to obtain some model-independent information of the scattering objects in the real space. ${ }^{39}$ The IFT of scattering data yields a pair distance correlation function $(p(r))$, which provides the information about particle shape, the radius of gyration $\left(R_{\mathrm{g}}\right)$, and the maximum size $D_{\max }$ of the scattering objects $\left(p(r)\right.$ goes to zero at $\left.r=D_{\max }\right)$. In the next step, a model for protein-surfactant complexes is constructed depending on the result obtained from the IFT of scattering data. A model for a beads-on-a-string chain is adapted for the surfactant-induced denaturation process of BSA protein. According to this model, the micelles are organized in clusters, which constitute a random flight string of polypeptide chain with a fixed step size between the centers of micelles. ${ }^{22}$

In general, the differential scattering cross section per unit volume $(\mathrm{d} \Sigma / \mathrm{d} \Omega)$ as a function of $q$ for monodisperse interacting particles is given by

$$
\left(\frac{\mathrm{d} \Sigma}{\mathrm{d} \Omega}\right)(q)=N_{\mathrm{p}} P(q) S(q)+B
$$

where $N_{\mathrm{p}}$ is the particle number density, $P(q)$ and $S(q)$ are the form factor and structure factor, respectively, and $B$ is the incoherent background arising mainly because of the presence of hydrogen in different components. $P(q)$, the form factor of a micelle, is assumed to be a prolate ellipsoidal core-shell structure. $P(q)$ of the prolate ellipsoidal core-shell particle with semiminor axis $R$, axial anisotropy $\varepsilon$, and thickness $t$ can be written as

$$
P(q)=\left\langle F^{2}(q)\right\rangle=\int_{0}^{\pi / 2}\left[F\left(q, r_{1}, r_{2}\right)\right]^{2} \sin \beta \mathrm{d} \beta
$$

$$
\text { where } \begin{aligned}
F\left(q, r_{1}, r_{2}\right)= & {\left[\left(\rho_{\mathrm{c}}-\rho_{\text {shell }}\right) V_{1}\left\{\frac{3 j_{1}\left(q r_{1}\right)}{q r_{1}}\right\}+\left(\rho_{\text {shell }}-\rho_{\mathrm{s}}\right)\right.} \\
& \left.V_{2}\left\{\frac{3 j_{1}\left(q r_{2}\right)}{q r_{2}}\right\}\right]
\end{aligned}
$$

with $j_{1}(x)=\frac{(\sin x-x \cos x)}{x^{2}}, r_{1}=R\left(\sin ^{2} \beta+\varepsilon^{2} \cos ^{2} \beta\right)^{1 / 2}$, and $r_{2}=(R+t)\left(\sin ^{2} \beta+\varepsilon^{2} \cos ^{2} \beta\right)^{1 / 2}$ where $\rho_{\mathcal{o}} \rho_{\text {shell }}$, and $\rho_{\mathrm{s}}$ are, respectively, the scattering length densities of the particle core, shell, and solvent. $V_{1}\left(=4 \pi \varepsilon R^{3} / 3\right)$ and $V_{2}\left(=4 \pi \varepsilon(R+t)^{3} / 3\right)$ are the volumes of the core and the core along with the shell, respectively. $\beta$ is the angle between the directions of the semimajor axis and wave vector transfer and $j_{1}(x)$ is a firstorder spherical Bessel function.

The structure factor of the random flight model with a constant step size can be expressed as ${ }^{40}$

$$
\begin{aligned}
S(q)= & {\left[\frac{2}{1-\sin (q D) /(q D)}-1\right.} \\
& \left.-\frac{2\left[1-(\sin (q D) /(q D))^{N_{\mathrm{CLU}}}\right]}{N_{\mathrm{CLU}}(1-\sin (q D) /(q D))^{2}} \frac{\sin (q D)}{q D}\right]
\end{aligned}
$$

Here, $N_{\text {CLU }}$ is the random flight steps or particularly the number of micelles per cluster and $D$ is the step size or the distance between the centers of two micelles. $N_{\text {CLU }}$ is calculated in the same way as mentioned by Giehm et al. ${ }^{22}$ as the weighted linear combination $\left[N_{\mathrm{CLU}}\right]$ and $\left[N_{\mathrm{CLU}}\right]+1$, with the $\left[N_{\mathrm{CLU}}\right]$ largest integer smaller than $N_{\mathrm{CLU}}$. 
By combining eqs $1-3$ and using the decoupling approximation, the differential scattering cross section per unit volume $(\mathrm{d} \Sigma / \mathrm{d} \Omega)$ for micelles with random flight structure can be expressed as ${ }^{31}$

$$
\left(\frac{\mathrm{d} \Sigma}{\mathrm{d} \Omega}\right)(q)=N_{\mathrm{mic}}\left[\left\langle F^{2}(q)\right\rangle+\langle F(q)\rangle^{2}(S(q)-1)\right]+B
$$

where $N_{\text {mic }}$ is the number density of micelles and calculated by $N_{\text {mic }}=N_{\mathrm{A}} \times\left(C_{\text {tot }}-C_{\mathrm{CAC}}\right) / N_{\text {agg. }} . N_{\mathrm{A}}$ is the Avogadro number, $C_{\text {tot }}$ is the total surfactant concentration, and $C_{\mathrm{CAC}}$ is the CAC of the surfactant. $N_{\mathrm{agg}}$ is the aggregation number of the micelle and determined by ratio of the volumes of the micellar core to the tail length of the surfactant. The volume of the tail length of the surfactant is calculated from Tanford's formula. ${ }^{41}$ CAC is an important parameter to get the exact number of micelles formed by the protein-surfactant complexes, which is determined by the DLS measurement. ${ }^{15}$ The concentration below CAC is assumed not to participate in the micelle formation. The information about the micelle size $(R)$, axial anisotropy in the size of the micelle $(\varepsilon)$, thickness $(t)$ of the micelle shell, and scattering length density of the shell $\left(\rho_{\text {shell }}\right)$ are extracted from the $P(q)$ of SANS data fitting. The scattering length density of core $\rho_{\mathrm{c}}$ is taken as the scattering length density of the hydrophobic tail of ionic surfactants $\left(-0.23 \times 10^{10} \mathrm{~cm}^{-2}\right)$, and $\rho_{\mathrm{s}}$ is the scattering length density of $\mathrm{D}_{2} \mathrm{O}\left(6.37 \times 10^{10} \mathrm{~cm}^{-2}\right)$. The independence of the fitted parameters is verified by the fitting of $q$-dependence of data and comparing scattering intensity on the absolute unit. The fitting of SANS data by the random flight structure factor gives the information about the number of micelles per cluster $\left(N_{\mathrm{CLU}}\right)$ and separation between two nearest micelles $(D)$. The number of proteins per micelle $\left(N_{\text {prot } / \text { mic }}\right)$ is calculated from the known protein concentration divided by $N_{\text {mic }}$. The product of the number of proteins per micelle and $N_{\text {CLU }}$ yields the number of proteins per cluster. All the data were fitted using SASfit analysis software. ${ }^{42}$

4.3. Dynamic Light Scattering. DLS experiments were carried out using an SZ-100 particle size analyzer (HORIBA, Japan) at a wavelength of $532 \mathrm{~nm}$. The scattering angle was kept fixed at $173^{\circ}$ during the measurements. All the samples used for DLS measurement were dispersed in $\mathrm{H}_{2} \mathrm{O}$ and measured without dilution. The backscatter detection ensures the minimization of any contamination from dust particles and multiple scattering from the sample. The DLS measures the temporal fluctuation in scattering light intensity at a specific angle using a monochromatic light. The signal generated by diffusing particles can be analyzed by the intensity autocorrelation function. The diffusion coefficient $\left(D_{\mathrm{a}}\right)$ and polydispersity index are calculated using the cumulant analysis method. The effective hydrodynamic size $\left(d_{\mathrm{h}}\right)$ is calculated from $D_{\mathrm{a}}$ using the Stoke-Einstein equation ${ }^{35}$

$$
d_{\mathrm{h}}=\frac{k_{\mathrm{B}} T}{3 \pi \eta D_{\mathrm{a}}}
$$

where $k_{\mathrm{B}}$ is Boltzmann's constant, $\eta$ is the viscosity of the solvent, and $T$ is the absolute temperature. The details of DLS theory are presented in the Supporting Information.

\section{ASSOCIATED CONTENT}

\section{S Supporting Information}

The Supporting Information is available free of charge on the ACS Publications website at DOI: 10.1021/acsomega.8b00630.

Details of theory of DLS, SANS analysis of form factor and structure factor of pure components, scattering length density calculation, IFT fitting of SANS profile of BSA with ionic surfactants, DLS results of unfolding of $\mathrm{BSA}$, and refolding of BSA protein in the presence of the nonionic surfactant studied by SANS and DLS (PDF)

\section{AUTHOR INFORMATION}

\section{Corresponding Author}

*E-mail: vkaswal@barc.gov.in. Phone: +91 22 25594642. Fax: +912225505150 (V.K.A.).

\section{ORCID}

Debasish Saha: 0000-0001-8298-8291

Debes Ray: 0000-0001-5564-2973

Vinod Kumar Aswal: 0000-0002-2020-9026

\section{Notes}

The authors declare no competing financial interest.

\section{ACKNOWLEDGMENTS}

D.S. would like to thank the Department of Science and Technology (DST), India, for the grant (DST/INSPIRE/04/ 2015/003265).

\section{REFERENCES}

(1) Sun, C.; Yang, J.; Wu, X.; Huang, X.; Wang, F.; Liu, S. Unfolding and Refolding of Bovine Serum Albumin Induced by Cetylpyridinium Bromide. Biophys. J. 2005, 88, 3518-3524.

(2) De, S.; Girigoswami, A.; Das, S. Fluorescence probing of albumin-surfactant interaction. J. Colloid Interface Sci. 2005, 285, $562-573$.

(3) El Kadi, N.; Taulier, N.; Le Huérou, J. Y.; Gindre, M.; Urbach, W.; Nwigwe, I.; Kahn, P. C.; Waks, M. Unfolding and Refolding of Bovine Serum Albumin at Acid $\mathrm{pH}$ : Ultrasound and Structural Studies. Biophys. J. 2006, 91, 3397-3404.

(4) Jones, M. N. Surfactant Interactions with Biomembranes and Proteins. Chem. Soc. Rev. 1992, 21, 127-136.

(5) Narayanan, J.; Deotare, V. W. Salt-Induced Liquid-Liquid Phase Separation of Protein-Surfactant Complexes. Phys. Rev. E: Stat. Phys., Plasmas, Fluids, Relat. Interdiscip. Top. 1999, 60, 4597-4603.

(6) Otzen, D. Protein-surfactant interactions: A tale of many states. Biochim. Biophys. Acta BBA - Proteins Proteomics 2011, 1814, 562591.

(7) Andersen, K. K.; Oliveira, C. L.; Larsen, K. L.; Poulsen, F. M.; Callisen, T. H.; Westh, P.; Pedersen, J. S.; Otzen, D. The Role of Decorated SDS Micelles in Sub-CMC Protein Denaturation and Association. J. Mol. Biol. 2009, 391, 207-226.

(8) Dobson, C. M. Protein Folding and Misfolding. Nature 2003, 426, 884-890.

(9) Chodankar, S.; Aswal, V. K.; Kohlbrecher, J.; Vavrin, R.; Wagh, A. G. Surfactant-Induced Protein Unfolding as Studied by SmallAngle Neutron Scattering and Dynamic Light Scattering. J. Phys. Condens. Matter 2007, 19, 326102.

(10) Chodankar, S.; Aswal, V. K.; Hassan, P. A.; Wagh, A. G. Structure of protein-surfactant complexes as studied by small-angle neutron scattering and dynamic light scattering. Phys. B Condens. Matter 2007, 398, 112-117.

(11) Mehan, S.; Aswal, V. K.; Kohlbrecher, J. Tuning of ProteinSurfactant Interaction to Modify the Resultant Structure. Phys. Rev. E: Stat., Nonlinear, Soft Matter Phys. 2015, 92, 32713. 
(12) Otzen, D. E.; Sehgal, P.; Westh, P. $\alpha$-Lactalbumin is unfolded by all classes of surfactants but by different mechanisms. J. Colloid Interface Sci. 2009, 329, 273-283.

(13) Kaspersen, J. D.; Søndergaard, A.; Madsen, D. J.; Otzen, D. E.; Pedersen, J. S. Refolding of SDS-Unfolded Proteins by Nonionic Surfactants. Biophys. J. 2017, 112, 1609-1620.

(14) Gelamo, E. L.; Itri, R.; Alonso, A.; da Silva, J. V.; Tabak, M. Small-angle $\mathrm{X}$-ray scattering and electron paramagnetic resonance study of the interaction of bovine serum albumin with ionic surfactants. J. Colloid Interface Sci. 2004, 277, 471-482.

(15) Chakraborty, T.; Chakraborty, I.; Moulik, S. P.; Ghosh, S. Physicochemical and Conformational Studies on BSA-Surfactant Interaction in Aqueous Medium. Langmuir 2009, 25, 3062-3074.

(16) Kelley, D.; McClements, D. J. Interactions of Bovine Serum Albumin with Ionic Surfactants in Aqueous Solutions. Food Hydrocoll. 2003, 17, 73-85.

(17) Valstar, A.; Almgren, M.; Brown, W.; Vasilescu, M. The Interaction of Bovine Serum Albumin with Surfactants Studied by Light Scattering. Langmuir 2000, 16, 922-927.

(18) Otzen, D. E. Protein Unfolding in Detergents: Effect of Micelle Structure, Ionic Strength, $\mathrm{pH}$, and Temperature. Biophys. J. 2002, 83, 2219-2230.

(19) Jelińska, A.; Zagożdżon, A.; Górecki, M.; Wisniewska, A.; Frelek, J.; Holyst, R. Denaturation of Proteins by Surfactants Studied by the Taylor Dispersion Analysis. PLoS One 2017, 12, e0175838.

(20) Jain, N.; Trabelsi, S.; Guillot, S.; McLoughlin, D.; Langevin, D.; Letellier, P.; Turmine, M. Critical Aggregation Concentration in Mixed Solutions of Anionic Polyelectrolytes and Cationic Surfactants. Langmuir 2004, 20, 8496-8503.

(21) Mallamace, F.; Corsaro, C.; Mallamace, D.; Vasi, S.; Vasi, C.; Baglioni, P.; Buldyrev, S. V.; Chen, S.-H.; Stanley, H. E. Energy Landscape in Protein Folding and Unfolding. Proc. Natl. Acad. Sci. U.S.A. 2016, 113, 3159-3163.

(22) Giehm, L.; Oliveira, C. L. P.; Christiansen, G.; Pedersen, J. S.; Otzen, D. E. SDS-Induced Fibrillation of $\alpha$-Synuclein: An Alternative Fibrillation Pathway. J. Mol. Biol. 2010, 401, 115-133.

(23) Sun, Y.; Filho, P. L. O.; Bozelli, J. C.; Carvalho, J.; Schreier, S.; Oliveira, C. L. P. Unfolding and Folding Pathway of Lysozyme Induced by Sodium Dodecyl Sulfate. Soft Matter 2015, 11, 77697777.

(24) Chen, S.-H.; Teixeira, J. Structure and Fractal Dimension of Protein-Detergent Complexes. Phys. Rev. Lett. 1986, 57, 2583-2586.

(25) Guo, X. H.; Zhao, N. M.; Chen, S. H.; Teixeira, J. Small-angle neutron scattering study of the structure of protein / detergent complexes. Biopolymers 1990, 29, 335-346.

(26) Anand, U.; Mukherjee, S. Reversibility in protein folding: effect of $\beta$-cyclodextrin on bovine serum albumin unfolded by sodium dodecyl sulphate. Phys. Chem. Chem. Phys. 2013, 15, 9375-9383.

(27) Gull, N.; Khan, J. M.; Rukhsana; Khan, R. H. Spectroscopic Studies on the Gemini Surfactant Mediated Refolding of Human Serum Albumin. Int. J. Biol. Macromol. 2017, 102, 331-335.

(28) Anand, U.; Jash, C.; Mukherjee, S. Protein Unfolding and Subsequent Refolding: A Spectroscopic Investigation. Phys. Chem. Chem. Phys. 2011, 13, 20418-20426.

(29) Sanchez-Fernandez, A.; Arnold, T.; Jackson, A. J.; Fussell, S. L.; Heenan, R. K.; Campbell, R. A.; Edler, K. J. Micellization of alkyltrimethylammonium bromide surfactants in choline chloride:glycerol deep eutectic solvent. Phys. Chem. Chem. Phys. 2016, 18, $33240-33249$.

(30) Zhang, F.; Roosen-Runge, F.; Skoda, M. W. A.; Jacobs, R. M. J.; Wolf, M.; Callow, P.; Frielinghaus, H.; Pipich, V.; Prévost, S.; Schreiber, F. Hydration and Interactions in Protein Solutions Containing Concentrated Electrolytes Studied by Small-Angle Scattering. Phys. Chem. Chem. Phys. 2012, 14, 2483-2493.

(31) Pedersen, J. S. Analysis of Small-Angle Scattering Data from Colloids and Polymer Solutions: Modeling and Least-Squares Fitting. Adv. Colloid Interface Sci. 1997, 70, 171-210.
(32) Franklin, J. M.; Surampudi, L. N.; Ashbaugh, H. S.; Pozzo, D. C. Numerical Validation of IFT in the Analysis of Protein-Surfactant Complexes with SAXS and SANS. Langmuir 2012, 28, 12593-12600.

(33) Svergun, D. I.; Koch, M. H. J. Small-Angle Scattering Studies of Biological Macromolecules in Solution. Rep. Prog. Phys. 2003, 66, $1735-1782$.

(34) Pecora, R. Dynamic Light Scattering Applications of Photon Correlation Spectroscopy; Springer US, 1985.

(35) Lindner, P.; Zemb, T. Neutrons, X-rays and Light: Scattering Methods Applied to Soft Condensed Matter, 1st ed.; North-Holland Delta Series; North-Holland: Netherlands, 2002; pp 203-220.

(36) Mehan, S.; Chinchalikar, A. J.; Kumar, S.; Aswal, V. K.; Schweins, R. Small-Angle Neutron Scattering Study of Structure and Interaction of Nanoparticle, Protein, and Surfactant Complexes. Langmuir 2013, 29, 11290-11299.

(37) Kohlbrecher, J.; Wagner, W. The New SANS Instrument at the Swiss Spallation Source SINQ. J. Appl. Crystallogr. 2000, 33, 804806.

(38) Keiderling, U. The new 'BerSANS-PC' software for reduction and treatment of small angle neutron scattering data. Appl. Phys. A 2002, 74, s1455-s1457.

(39) Oliveira, C. L. P.; Behrens, M. A.; Pedersen, J. S.; Erlacher, K.; Otzen, D.; Pedersen, J. S. A SAXS Study of Glucagon Fibrillation. J. Mol. Biol. 2009, 387, 147-161.

(40) Burchard, W.; Kajiwara, K. The Statistics of Stiff Chain Molecules. I. The Particle Scattering Factor. Proc. R. Soc. London, Ser. A 1970, 316, 185-199.

(41) Tanford, C. The Hydrophobic Effect. Formation of Micelles and Biological Membranes, 2nd ed.; Wiley and Sons: New York, 1980.

(42) Bressler, I.; Kohlbrecher, J.; Thünemann, A. F. SASfit: A Tool for Small-Angle Scattering Data Analysis Using a Library of Analytical Expressions. J. Appl. Crystallogr. 2015, 48, 1587-1598. 